\title{
Attribute-guided well-log interpolation applied to low-frequency impedance estimation
}

\author{
Thomas Mejer Hansen ${ }^{1}$, Klaus Mosegaard ${ }^{1}$, Radmila Pedersen-Tatalovic ${ }^{2}$, \\ Anette Uldall ${ }^{2}$, and Nils Lange Jacobsen ${ }^{2}$
}

\begin{abstract}
Several approaches exist to use trends in 3D seismic data, in the form of seismic attributes, to interpolate sparsely sampled well-log measurements between well locations. Kriging and neural networks are two such approaches. We have applied a method that finds a relation between seismic attributes (such as two-way times, interval velocities, reflector roughness) and rock properties (in this case, acoustic impedance) from information at well locations. The relation is designed for optimum prediction of acoustic impedances away from well sites, and this is accomplished through a combination of cross validation and the Tikhonov-regularized least-squares method. The method is fast, works well even for highly underdetermined problems, and has general applicability. We apply it to two case studies in which we estimate 3D cubes of low-frequency impedance, which is essential for producing good porosity models. We show that the method is superior to traditional least squares: Numerous blind tests show that estimated low-frequency impedance away from well locations can be determined with an accuracy very close to estimations obtained at well locations.
\end{abstract}

\section{INTRODUCTION}

Oil exploration typically exploits a combination of boreholes and seismic surveys. Boreholes provide direct measurements of the subsurface, and 3D seismic surveys provide dense indirect measurements of elastic properties over a large area. Joint inversion of the full seismic wavefield and well-log data is not feasible at this time. This is one reason it has become popular to extract certain features from the seismic data, called seismic attributes. For instance, a geologic horizon (defined by the two-way traveltime to a layer interface) can be correlated to well logs and tracked throughout the 3D volume of seismic data. In addition to two-way traveltime maps, many horizon- or volume-based attributes can be extracted from the 3D seismic volume. See Chopra and Marfurt (2005) for a comprehensive list of available seismic attributes.

Consider an area where wells provide good knowledge of the subsurface at single points. In subsurface prediction, often it is desirable to extrapolate such measurements away from well locations; in some cases, additional surface measurements are available to assist. Each well log samples the geologic setting at the specific well location, but little is known about variations between the wells. The task now is to estimate variations of the logged physical/geologic properties away from the boreholes, using surface measurements as a guide. This is a typical problem in many geophysical settings.

A typical geophysical problem is the following: Based on observed data $\mathbf{d}$, estimate the model $\mathbf{m}$ that, given some physical relationship $\mathbf{d}=\mathbf{G m}$, optimally would predict $\mathbf{d}$. This is a typical inverse problem that has been studied widely; see, for example, Tarantola (1987). In this paper, we address the less frequent situation of measuring corresponding sets of $\mathbf{d}$ and $\mathbf{m}$, but when no physical relation $\mathbf{d}=\mathbf{G m}$ is known that maps $\mathbf{m}$ into $\mathbf{d}$. The problem is to estimate the transfer function $\mathbf{G}$.

The method described here was developed to solve a specific problem in seismic reservoir characterization, which is to estimate the low-frequency impedance (product of the P-wave velocity and density) using 3D reflection seismic data and well-log data.

Seismic reflection data are sensitive to very low frequencies of the velocity field, less than about $2 \mathrm{~Hz}$, and relatively high frequencies of the impedance, the so-called reflectivity, in the range of about 8-70 Hz. There is, however, no sensitivity of the seismic P-wave reflection wavefield to the intermediate frequency range of about $2-8 \mathrm{~Hz}$; see Figure 1. This area of no resolution is known as the information gap (Claerbout, 1985; Jannane et al., 1989).

Neves and Singh (1996) show that long-offset pressure and converted-wave data are sensitive to frequencies in the information-gap

Manuscript received by the Editor 4 August 2007; revised manuscript received 11 June 2008; published online 14 November 2008.

${ }_{1}^{1}$ University of Copenhagen, Niels Bohr Institute, Copenhagen, Denmark.E-mail: tmh@ gfy.ku.dk; cultpenguin@gmail.com; klaus@gfy.ku.dk.

${ }^{2}$ Mærsk Oil and Gas, Copenhagen, Denmark.E-mail: rpt@maerskoil.com; au@ maerskoil.com; njacobsen@maerskoil.co.uk.

(C) 2008 Society of Exploration Geophysicists. All rights reserved. 

wave data, recorded at relatively short offsets (well below the critical angle). Therefore, methods are needed for recovering the missing low-frequency information.

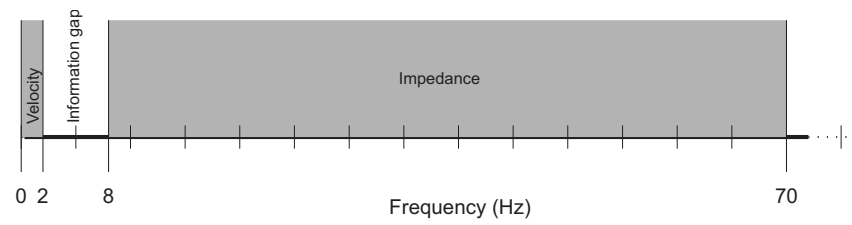

Figure 1. Conventional reflection seismic data are sensitive (indicated by gray) to very long wavelengths, i.e., low frequencies $(<2 \mathrm{~Hz})$ of the velocity field, and the relative short wavelengths, i.e., high frequencies $(>8 \mathrm{~Hz},<70 \mathrm{~Hz})$, of the impedance field. The information gap is the intermediate frequency range from about $2 \mathrm{~Hz}$ through $8 \mathrm{~Hz}$ not sampled by conventional reflection seismic data. range. Yet, most recorded seismic data have been, and are, pressure-

Figure 2 shows the relative impedance, low-frequency component of the impedance, and the full impedance log for three wells. The full $\log$ and the low-frequency filtered log show the slow buildup of the impedance. The relative impedance log, which is obtained by filtering out the low-frequency component from the full impedance $\log$, shows only the relatively high-frequency variations; the absolute values of the impedance are unknown. The low-frequency part of the well log is essential for reconstruction of these values.

In this example, aimed at analyzing the porosity of chalk of the North Sea, there exists a very good linear relation between impedance and porosity. Critical to the quality of the seismic-porosity estimate is correct modeling of the absolute impedance. Figure 3 shows two cross plots of an impedance log versus a porosity log, without (Figure 3a) and with (Figure 3b) the low frequencies being retained in the impedance log. It is obvious that without the low frequencies, the strong correlation is lost. Therefore, without the low frequencies, no reliable estimate of porosity can be made.

Because seismic data show no sensitivity to the $2-8 \mathrm{~Hz}$ impedance frequency range, a technique other than waveform inversion must be used to recover this information. In the a)

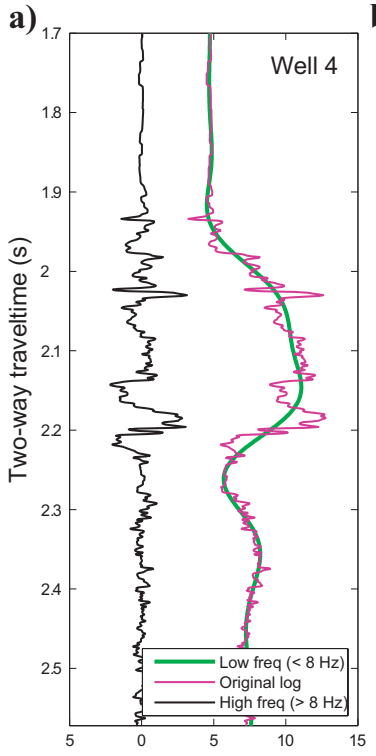

b)

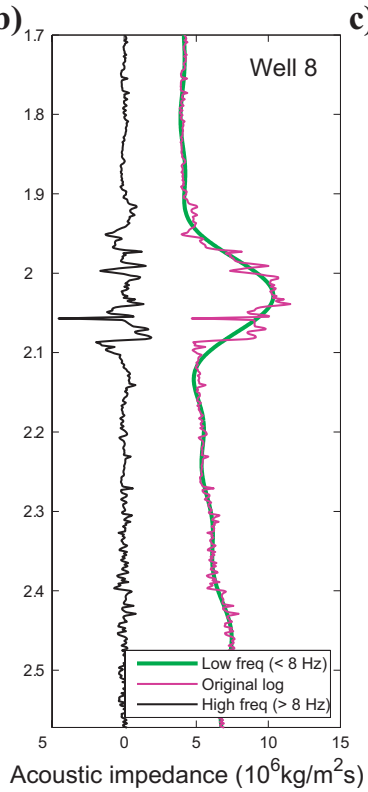

c)

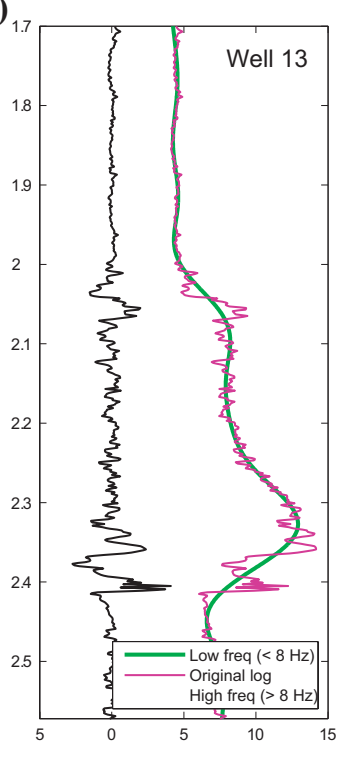

Figure 2. Relative acoustic impedance (AI), $>8 \mathrm{~Hz}$ (black solid), original log (purple), and the low-frequency part of the original $\log$ (green) for wells 4,8 , and 13.
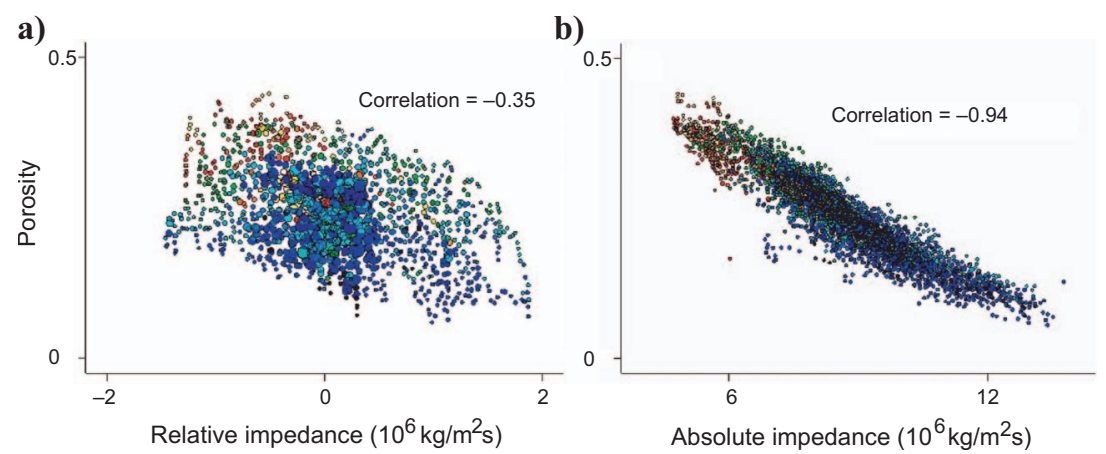

Figure 3. Cross plot of impedance and porosity logs, color coded with water saturation: Warm colors indicate low water saturation. (a) Using band-pass-filtered logs approximating seismic bandwidth. (b) Using the full frequency range. following, we refer to frequencies below $8 \mathrm{~Hz}$ as "low frequencies."

We present a solution to the general problem of extrapolating/interpolating properties of point measurements (for instance, well logs) using indirect observations in the form of regionally defined geophysical attributes. The method is probabilistic and based on a combination of leastsquares inversion using Tikhonov regularization and cross validation. We apply the methodology to the problem of 3D interpolation of low-frequency impedance between well locations, guided by seismic attributes.

\section{EXISTING EXTRAPOLATION TECHNIQUES}

In the following, we consider the general problem of interpolation and extrapolation of point data, but for the sake of clarity, we concentrate on seismic attribute-guided interpolation of borehole information. We consider two scenarios: (1) only point measurements are available, i.e., univariate data; and (2) point data and area-covering measurements, such as seismic or other types of geophysical data, are available, i.e., multivariate data.

\section{Univariate data}

Techniques using distance, spline, nearestneighbor, and kriging can be applied when only point measurements are available. Kriging-based interpolation relies on a model of the spatial correlation between pairs of data. The method requires definition of a covariance model estimated from available data (Goovaerts, 1997). If only few data are available, or if large variations in geology occur, it could be difficult to produce a covariance model. If only very few data are avail- 
able, triangular interpolation or nearest-neighbor interpolation might be the best choice.

Billings et al. (2002) discuss the use of continuous global surfaces as a general approach for univariate interpolation.

\section{Multivariate data}

When point data and area-covering data are available, several more sophisticated approaches exist. Here, we briefly recall methods based on linear regression, geostatistics, and neural networks.

Multivariate linear regression is a simple method for inferring a linear trend (defined by a linear operator) in a multivariate data set. Using least-squares regression, the linear operator is found by minimizing the least-squares prediction error. See Hampson et al. (2001) for an introduction related to analysis of seismic attributes.

Kriging can be extended to multivariate data interpolation, the socalled cokriging method. This extension is straightforward but rather complex to use. Instead of using one covariance model to describe the spatial correlation, one must determine some covariance and crosscovariance models. Having just one additional variable, two covariance models and one crosscovariance model ideally should be determined from the observed data. For two additional variables, three covariance and three crosscovariance models must be determined. Thus ordinary cokriging becomes relatively complex and impractical to use, even with few data.

Certain variations of cokriging overcome, to some extent, the problem of estimating multivariate covariance matrices. In oil exploration, cokriging has been applied, for example, by Doyen (1988) and collocated cokriging by Xu et al. (1992).

Kriging and cokriging techniques perform very well in some cases, but they suffer from at least three problems: (1) Kriging relies on the fundamental assumption that data correlations depend on only distance and direction in physical space. This is not always a good assumption in interpolation: Two nearby points on opposite sides of a fault might give very different data, so it is not trivial to model faults with kriging techniques. (2) A minimum number of data points are required to select a proper covariance model. Even if enough data are available, it can be a tedious task to infer the covariance and crosscovariance models. (3) The covariance model reflects the variation around a trend model that is unknown, except in very simple cases. See, for instance, Goovaerts (1997) for an introduction to multivariate kriging techniques.

Neural networks have gained significant attention in geophysical literature in recent years (Liu and Liu, 1998; Kalkomey, 1997; Leiphart and Hart, 2001; Hampson et al., 2001; Banchs and Michelena, 2002; Poulton, 2002; Russell et al., 2002; Pramanik et al., 2004; Herrera et al., 2006). Neural networks provide a convenient framework for dealing with strongly nonlinear problems. A neural network can be used to approximate any continuous, nonlinear function with arbitrary accuracy, if an infinite number of suitable data are present (Bishop, 1995). However, an infinite number of data are never available, and therefore the application of a neural network usually involves dividing the data into a training set and a validation set for validating the performance of the network.

Hampson et al. (2001) compare linear regression for seismicattribute analysis to two implementations of neural networks, a multilayer feed-forward neural network (MLFN) and a probabilistic neural network (PNN). A PNN is essentially an interpolation technique using kernel functions. Hampson et al. (2001) demonstrate that MLFN can be overtrained easily, whereas PNN gives a more stable result. The amount of smoothness of the PNN interpolation is controlled by the number of attributes, cross validation, and shape of the kernels used. Leiphart and Hart (2001) implement a PNN and compare its result with multiple linear-attribute regression. In these cases, and in Hampson et al. (2001), the number of (independent) well-log data is significantly higher than the number of seismic attributes being used; i.e., the learning problem is overdetermined.

An important aspect using any kind of neural network is feature extraction, whereby selecting the most significant attributes reduces the dimension of the problem. Practically, it is impossible to include all types of attributes because this would require a large amount of training data and processing time. Trappe and Hellmich (2000), Liu and Liu (1998), and Hampson et al. (2001) use a small fraction of the available seismic attributes.

For an introduction to neural networks, see, for instance, Bishop (1995). For a review paper on the use of neural networks in geophysics, see Poulton (2002). For the specific application of estimating a low-frequency impedance model, see Nivlet (2004), who estimates an initial low-frequency impedance model directly from normal moveout (NMO) velocities. This low-frequency model then is calibrated to well-log data using linear regression and used as a trend model for factorial kriging to produce a low-frequency model of the subsurface.

We present an alternative to these methods for multivariate interpolation, based on a combination of cross validation and leastsquares inversion. This alternative has new features that overcome some limitations of existing methods: It works for highly underdetermined problems, is easy to apply, honors geologic subsurface structures (such as faults), and is robust with respect to overtraining caused by use of cross validation.

\section{THEORY}

The theory applies to the specific problem of estimation of a 3D low-frequency impedance cube using a 3D seismic data set and some impedance well logs. We use the term "parameterization" as the process of characterizing a geologic model by a series of real numbers, for instance, approximation of the geologic model by a simple, stratified model. The term 'deparameterization' is defined as the (reverse) process of "assembling" a complete 3D cube of data from a parameterized model through appropriate interpolation.

Parameterization and deparameterization (as described in the following) are specific to this problem, but the learning and prediction parts apply to any interpolation and/or extrapolation of point measurements using indirect multivariate area-covering data.

Consider a collection of $N$ wells, where each well is characterized by seismic information $m$ and acoustic impedance $d$. The symbols $m$ and $d$ typically stand for either vectors or functions of depth. We assume that $m$ depends on $d$, but no mathematical expression $m=f(d)$ relating $d$ and $m$ is available. The only information we have is corresponding pairs of $d$ and $m$ for all wells: $\left(d_{1}, m_{1}\right),\left(d_{2}, m_{2}\right), \ldots,\left(d_{N}, m_{N}\right)$. 
The problem is to find (or "learn") the relationship between $d$ and $m$, and to use this knowledge to estimate the acoustic impedance $d$ from seismic information $m$, or vice versa.

The present method solves this problem through a probabilistic approach in which the pairs $\left(d_{1}, m_{1}\right),\left(d_{2}, m_{2}\right), \ldots,\left(d_{N}, m_{N}\right)$ are "fitted" by a probability density, rather than by a smooth function. In this way, we can interpolate between the $(d, m)$ pairs, and produce uncertainty estimates of the predictions.

The problem is set as follows: For each point in a geographic grid, seismic data $m$ are given as vectors $\mathbf{m}^{\prime}$ of sampled values. Well-impedance information $d$ is available in a few $($ say, $N)$ of these points as vectors d' of sample values.

\section{Data and parameterization}

The first step is to parameterize the problem. Some geologic horizons are identified and interpreted as lithologic/formation changes, typically related to impedance contrasts. Each horizon normally is represented as a map of two-way traveltime. For each such horizon, seismic data and well-log data are "sparsely" represented in the following way:

Seismic data $\mathbf{m}^{\prime}$ are represented by event vectors $\mathbf{m}$ (at every geographic position), whose components are, for example: (1) two-way time from surface to selected seismic markers (each giving rise to conspicuous reflections); (2) reflection amplitudes for each marker; (3) interval velocity for geologic layers; and (4) texture attributes as, for example, measures of coherence of seismic reflections between seismic traces. Other seismic attributes are possible to the extent that they can be quantified.

Well data $\mathbf{d}^{\prime}$ are represented by event vectors $\mathbf{d}$ containing lowpass-filtered (approximately $0-8 \mathrm{~Hz}$ ) versions of $\mathbf{d}^{\prime}$, sampled exactly at the depth (two-way time) defined by two-way traveltime maps at the well locations.

Thus, for each geologically defined horizon, the goal is to find the function $f$ that in some optimal way maps the attributes into low-frequency impedance, given the set of $N$ observed $(\mathbf{d}, \mathbf{m})$.

\section{Learning and prediction}

Consider the spaces $\mathcal{D}$ and $\mathcal{M}$ of possible vectors $\mathbf{d}$ and $\mathbf{m}$, respectively. The collection of points,

$$
\left(\mathbf{d}_{1}, \mathbf{m}_{1}\right),\left(\mathbf{d}_{2}, \mathbf{m}_{2}\right), \ldots,\left(\mathbf{d}_{N}, \mathbf{m}_{N}\right)
$$

will, if there is any function that maps $\mathbf{m}$ into $\mathbf{d}$, cluster around a submanifold in $\mathcal{D} \times \mathcal{M}$ of maximum dimension $\operatorname{Dim}(\mathcal{M})$. Furthermore, assuming that the collection of points (equation 1) is following a Gaussian distribution, it will cluster around an affine subspace $\mathcal{A}$ (a "shifted linear subspace") in $\mathcal{D} \times \mathcal{M}$. We also assume that the uncertainty of $\mathbf{d}$ and $\mathbf{m}$ can be described by a Gaussian function with mean 0 .

The first step in the approximate characterization is to find $\mathcal{A}$. To do that, we must define precisely what we mean by "clustering around an affine subspace." The intention is to interpret $\mathcal{A}$ as the graph of a linear function $\mathbf{G}$ that optimally predicts $\mathbf{d}$ from $\mathbf{m}$, through the equation

$$
\mathbf{d}=\mathbf{G}\left(\mathbf{m}-\mathbf{m}_{0}\right)+\mathbf{d}_{0},
$$

where $\mathbf{d}_{0}$ and $\mathbf{m}_{0}$ are average vectors of $\mathbf{d}$ and $\mathbf{m}$, respectively, over the existing wells.

One possible way of obtaining such a linear function (matrix) $\mathbf{G}$ is to determine the affine hyperplane $\mathcal{A}$ through a linear regression based on the points (equation 1). This will result in a hyperplane with optimal fit (in a least-squares sense) to the points. However, it will not lead, in general, to useful predictions of $\mathbf{d}$ from $\mathbf{m}$. The reason for this apparent paradox is that the points (equation 1) are noisy, in the sense that, at a given well site, local contributions to the relation (equation 2) exist, which cannot be found elsewhere. Such contributions must be ignored in the design of the prediction relation (equation 2) to ensure that predictions are reliable. In general, a trade-off exists between accuracy of fitting the well data (equation 1) and the reliability of predicting parameters $\mathbf{d}$ from $\mathbf{m}$ at new locations. In this approach, the trade-off is controlled by a Tikhonov regularization of the least-squares estimate of $\mathbf{G}$ (Tikhonov, 1977):

$$
\mathbf{G}_{e s t}=\left[\mathbf{W}_{m}^{-1} \mathbf{M}\left(\mathbf{M}^{T} \mathbf{W}_{m}^{-1} \mathbf{M}+\lambda^{2} \mathbf{W}_{d}^{-1}\right)^{-1} \mathbf{D}^{T}\right]^{T},
$$

where $\mathbf{M}$ is the matrix with column vectors $\mathbf{m}_{1}, \mathbf{m}_{2}, \ldots, \mathbf{m}_{N}$; $\mathbf{D}$ is the matrix with column vectors $\mathbf{d}_{1}, \mathbf{d}_{2}, \ldots, \mathbf{d}_{N}$; the matrices $\mathbf{W}_{m}$ and $\mathbf{W}_{d}$ are diagonal weight matrices for $\mathbf{m}$ and $\mathbf{d}$, respectively; and $\lambda$ is a damping parameter. The damping parameter controls the regularization. A small $\lambda$ indicates little regularization, whereas a large $\lambda$ indicates strong regularization.

The entities $\mathbf{W}_{m}, \mathbf{W}_{d}$, and $\lambda$ determine the trade-off between the ability of $\mathbf{G}_{e s t}$ to fit the well data and its ability to predict reliably. In the special case where $\mathbf{W}_{d}$ and $\mathbf{W}_{m}$ are identity matrices, and $\lambda=0$, the prediction operator $\mathbf{G}_{e s t}$ will fit the well data optimally, but the predictions away from the wells will be poor in general.

The actual values of $\mathbf{W}_{d}, \mathbf{W}_{m}$, and $\lambda$ used in the prediction are determined as the ones that minimize

$$
E_{t}=E+a Q
$$

where $E$ is the cross-validation error defined as

$$
\begin{aligned}
E= & \sum_{n=1}^{N}\left(\mathbf{d}_{n}-\left(\mathbf{G}_{e s t}^{k=1, \ldots, n-1, n+1 \ldots N}\left(\mathbf{W}_{d}, \mathbf{W}_{m}, \lambda\right)\right.\right. \\
& \left.\left.\times\left(\mathbf{m}_{n}-\mathbf{m}_{0}\right)+\mathbf{d}_{0}\right)\right)^{2}
\end{aligned}
$$

Here, $\mathbf{G}_{e s t}^{k=1, \ldots, n-1, n+1 \ldots N}\left(\mathbf{W}_{d}, \mathbf{W}_{m}, \lambda\right)$ is a prediction matrix calculated from all wells, except the $n$th well. Expression $E$ is a measure of the average ability of the prediction matrices to predict successfully unknown well data from known well data. Each $N$ well is contributing to $E$ by playing the role of an "unknown" well, this well being predicted from remaining $N-1$ wells, and contributing with a misfit between this prediction and the actual well data $\mathbf{d}_{n}$. 
Expression $Q$ is the standard deviation of the propagated noise and is calculated by propagating the noise of the input-well data $\mathbf{D}$ and $\mathbf{M}$ through equation 3 to the prediction matrix $\mathbf{G}_{e s t}$, using Monte Carlo simulations. The uncertainty of $\mathbf{G}_{e s t}$ follows directly from this. Then, using equation 2, the uncertainty of $\mathbf{G}_{e s t}$ and $\mathbf{m}$ (away from boreholes) can be propagated into a standard deviation of the uncertainties of predicted well data $\mathbf{d}$ at each grid point. Expression $a$ is a regularization constant, controlling to which degree the propagated uncertainty $Q$ will guide the inference of $\mathbf{W}_{d}, \mathbf{W}_{m}$, and $\lambda$.

What remains is to calculate the uncertainty of the estimated $\mathbf{d}_{e s t}$. In the present implementation, the variances are calculated as a sum of two contributions: The first contribution is the standard deviation of propagated noise distributions, as given by $Q$. The second contribution comes from the spread of the points (equation 1) around $\mathrm{A}$ and is a result of the choice of parameterization, which is too simple to account for all observed effects. This part is calculated using the $n$ individual well misfits that contribute to the sum in equation 5 for optimal values of $\mathbf{W}_{d}, \mathbf{W}_{m}$, and $\lambda$.

\section{Deparameterization}

A 3D cube of low-frequency impedances, $\mathbf{d}$, can be estimated by applying a 1D vertical interpolation between impedance estimates at the event horizons. Finally, a vertical low-pass filter - the same as used in the initial parameterization - is applied to ensure that data are in the correct low-frequency range.

\section{CASE I - LOW-FREQUENCY IMPEDANCE ESTIMATION}

We present a case study from the Danish North Sea, where we have used well logs and seismic data from the chalk section in a $30 \times 30-\mathrm{km}$ area, sampled in a $100 \times 100-\mathrm{m}$ grid. Figure $4 \mathrm{a}$ shows the two-way traveltime to the top chalk horizon, and the 13 well locations. The analysis and calculation procedure followed the same work flow as presented in the theory: parameterization, learning, prediction, and deparameterization.

\section{Parameterization}

The parameterization was simply a reorganization of available data to be used by the learning algorithm.

\section{Seismic events}

Five key geologic horizons were identified in the area and used as primary event horizons characterizing the chalk, namely top chalk, top Maastrichtian, top hod, intra chalk, and base chalk. In addition, eight secondary event horizons were introduced to allow an increase in the reconstructible frequency range. Four horizons were placed $50 \mathrm{~ms}$ and $100 \mathrm{~ms}$ above top chalk and below base chalk. Four horizons were placed between the five primary event horizons. These four hori- zons were considered only in areas where the thickness between event horizons was above $10 \mathrm{~ms}$. All in all, 13 were considered. We introduced as few secondary horizons as possible while ensuring that the resulting logs could be sampled adequately according to the low-pass filter of 8-Hz cutoff. A mapping function, $\mathbf{G}$, was estimated for each event horizon.

\section{The use of wells}

We used 13 wells with different penetration depth. Optimization was performed for one horizon at a time, using data from points of well penetration. This allowed us to use data from deviated wells and from wells that did not penetrate the full sequence. A deviated well, in principle, could cross a horizon several times, giving rise to more learning data for that horizon. The length of the event vector, or the number of low-frequency measurements at each horizon, could vary in this way from horizon to horizon.

Figure 2 shows the log response of three of the 13 wells from the test area. As indicated, only the low-frequency part of the impedance $\log$, the green line in Figure 2, was used in the calculation process, and a Butterworth low-pass filter with cutoff at $8 \mathrm{~Hz}$ was applied to the data. a)

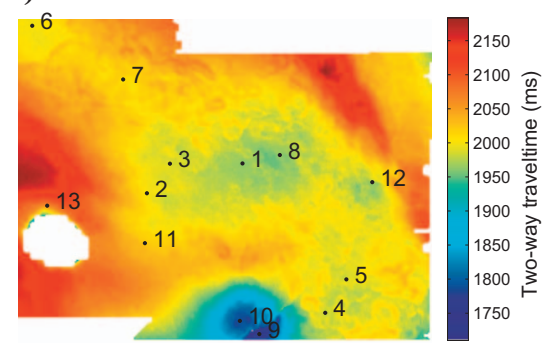

c)

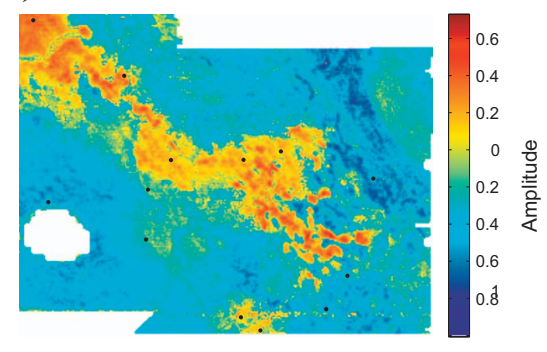

e)

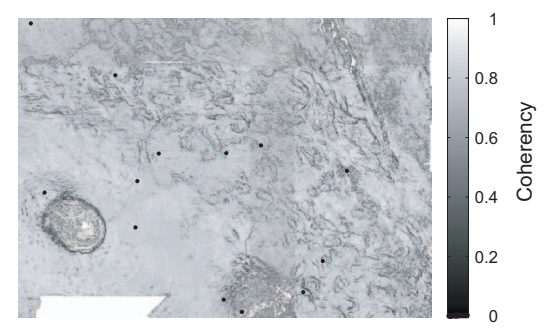

b)

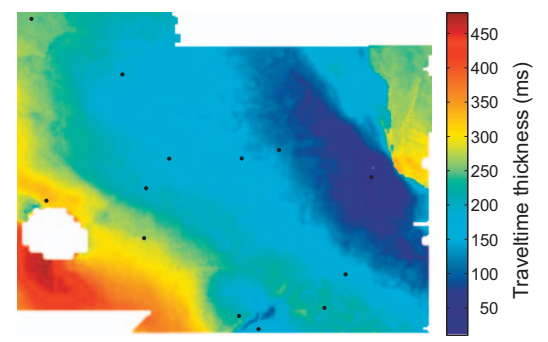

d)

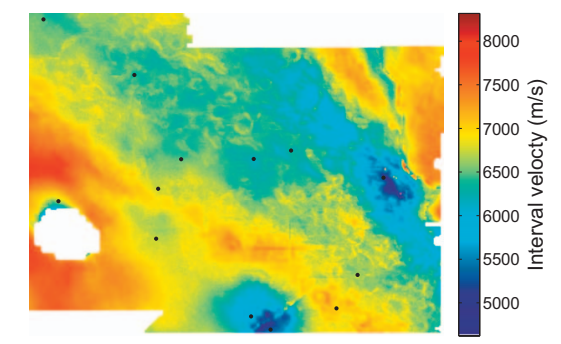

f)

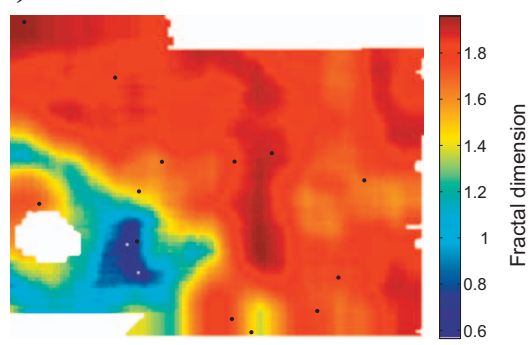

Figure 4. Seismic attribute types used for case study I: (a) Two-way traveltime to top chalk. (b) Traveltime thickness between top and base chalk. (c) Seismic amplitude at top chalk. (d) Interval velocity between top and base chalk. (e) Coherency at top chalk. (f) Fractal dimension at top chalk. 


\section{Attributes}

Seismic attributes can be divided naturally into three groups: (1) Continuous attributes could be two-way traveltime, seismic amplitude, seismic phase, volume attributes, and more. (2) Ordinal attributes are discrete with possible lithologic qualifiers, such as clay, sand, or gravel, but ordered (in terms of grain size, for example). (3) Nominal attributes are discrete and unordered, also called categorial data. Many seismic textures are of this type. An attribute with possible values onlap, offlap, or none is discrete and unordered. Classification maps belong to this class.

Continuous data can be used by the method just as they are. Ordinal data can be converted easily into a continuous attribute. To use nominal data, such as seismic textures, some methods exist to quantify observable patterns. See, e.g., Press et al. (1992) for variable types.

\section{Seismic attributes used in this study}

A 3D seismic data set was available for the test area. From these data, a large number of seismic attributes were generated (Brown, 2001; Chopra and Marfurt, 2005). We considered the following attributes:

Two-way traveltimes - Five maps of two-way traveltime (TWT) to the geologic primary event horizons were used directly as continuous attributes; see Figure 4a for one horizon. These TWT horizons also were used to calculate time thickness of individual layers, and to control the extraction of amplitude and coherence attributes using the seismic 3D volume.

Thickness - Ten time-thickness attributes, calculated from the five TWT maps, were used. See Figure $4 b$ for one example.

Amplitude - We used five maps of seismic-event amplitude, extracted along each of the five seismic TWT horizons. See Figure $4 \mathrm{c}$ for one example.

Interval velocity - One map of interval velocity in the chalk section, obtained by using horizon-consistent velocity analysis (HVA), was used. See Figure 4d. This is an important attribute because velocity is linked closely to impedance.

Coherency maps - Five maps of the coherence between neighboring seismic traces provided the coherency attributes. These are nominal attributes and therefore needed some preprocessing to be used. See Figure 4e for one example.

All considered seismic attributes carry geologic information and might correlate to the acoustic impedance. In general, the pressure will increase as the TWT increases. Therefore the density, and hence the acoustic impedance, will tend to increase. The amplitudes carry information about relative changes in impedance between sedimentary layers. Coherence maps might indicate fracture density. The velocity attribute obviously is linked closely to acoustic impedance.

\section{Using seismic textures}

Many seismic textures are nominal data, i.e., discrete and unordered, such as the coherency attribute in Figure 4e. For this attribute, the values are binary: Either there is coherence (1) or there is not (0). Therefore this attribute cannot be used directly in the numerical scheme. However, Figure 4e clearly indicates distinct geologic features: Some areas appear rougher than others. Fractal analysis is a tool that can be used to quantify such texture attributes, and in this case to quantify coherence in terms of fractal dimension of the roughness of the coherence plot.

We used the box counting method (see Gonzato et al., 2000) to extract the fractal dimension of the coherency maps. Figure $4 \mathrm{f}$ shows the estimated fractal dimension at intra chalk. The fractal dimension at a point was computed over a region (of size $1600 \times 1600$ meters), centered at the point, from the densely sampled coherency maps $(12.5 \times 12.5$ meters $)$. The final grid mesh is $100 \times 100 \mathrm{~m}$. Compared to Figure $4 \mathrm{f}$, it is apparent that the fractal dimension accurately identifies rough areas of the coherence map with a high fractal dimension and smooth areas of the map with a low fractal dimension.

Thus the parameterization of the problem was determined by the event data set, comprising five two-way traveltime maps, eight secondary two-way traveltime maps, and 13 wells with different penetration depth. The uncertainty (standard deviation of the Gaussian noise distribution) of the well-log impedance was set to $1 \%$ of the impedance value. All in all, 26 attributes were available. See Figure 4a through $4 \mathrm{f}$ for an example of each type of attribute. An absolute uncertainty of the TWT and thickness attribute was set to $3 \mathrm{~ms}$ and $4 \mathrm{~ms}$, respectively. The uncertainty of the amplitude attribute map was set to 100 amplitude units. The uncertainty of the interval velocity was set to $100 \mathrm{~m} / \mathrm{s}$, and the uncertainty of the fractal dimension maps was set to $15 \%$ of the fractal dimension value. The available length scales are limited, and it is impossible to obtain an exact fractal dimension (Gonzato et al., 2000).

For each event horizon, the learning and prediction process was performed as explained previously.

\section{Learning}

As discussed previously, an optimal mapping function $\mathbf{G}$ is determined for each of the 13 event horizons in the learning process. The case study illustrates how $\mathbf{G}$ is obtained for the top chalk event horizon. Wells 6 and 9 were not used, but they were included to investigate the performance of the presented method.

\section{Least-squares linear regression}

Initially, all attributes and data were weighted equally, and the mapping function $\mathbf{G}$ was calculated using all data and attributes, by using equation 3 . Without regularization $(\lambda=0)$, the calculation resembles standard linear regression (the least-squares solution) as used by Hampson et al. (2001), for example, to compare with neural network methods.

Figure 5a shows the true and predicted impedance at wells penetrating the top chalk horizon. Because the number of attributes was larger than the number of well data, and no regularization was applied, the estimated impedance at well locations equaled the true impedance: The prediction error was zero. On the other hand, the crossvalidation error was very large, indicating that the prediction operator $\mathbf{G}$ worked well only at well locations. The mean prediction and cross-validation errors are listed in Table 1.

Similar results for a deeper horizon, intra chalk (where fewer well data are available because of the limited penetration depth of several wells), are listed in Table 2. Although the low prediction er- 
ror at blind wells indicates that the prediction is reasonable, the cross-validation error clearly shows that this conclusion is wrong. The corresponding prediction at the top chalk horizon is shown in Figure $6 a$, whereas the prediction of the low-frequency impedance at the intra chalk horizon is shown in Figure 7a.

\section{Least-squares linear regression with feature extraction}

Feature extraction is used widely to reduce the dimension of the attribute space, to improve computational efficiency, and to provide more stable results; see, e.g., Hampson et al. (2001). Feature extrac- a)

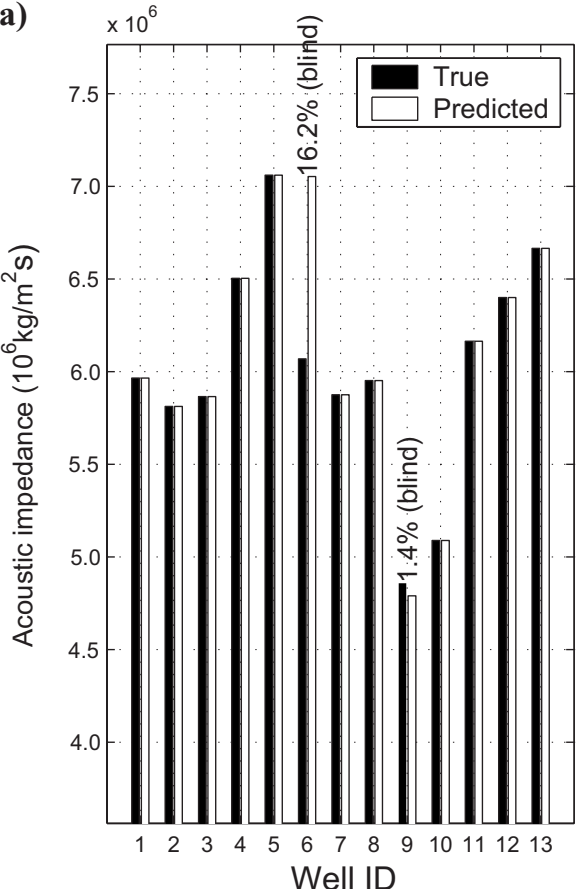

b)

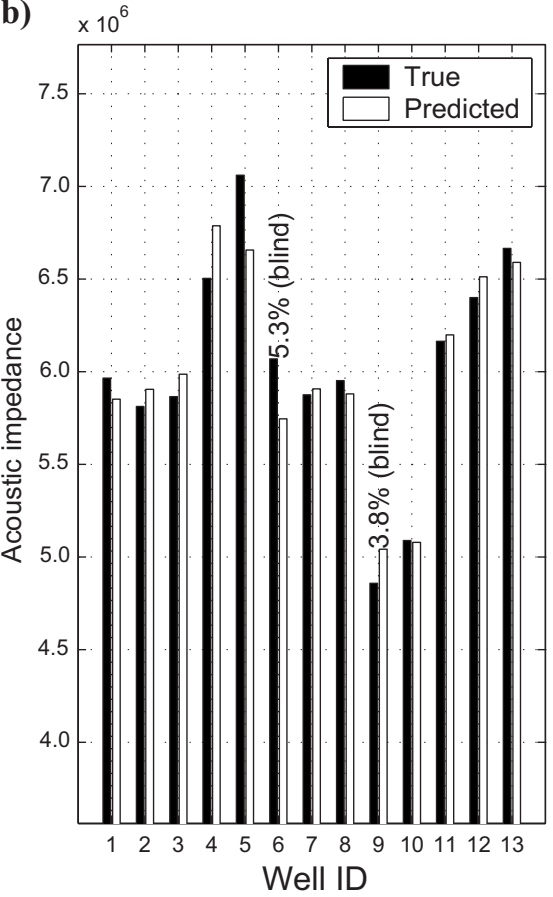

c)

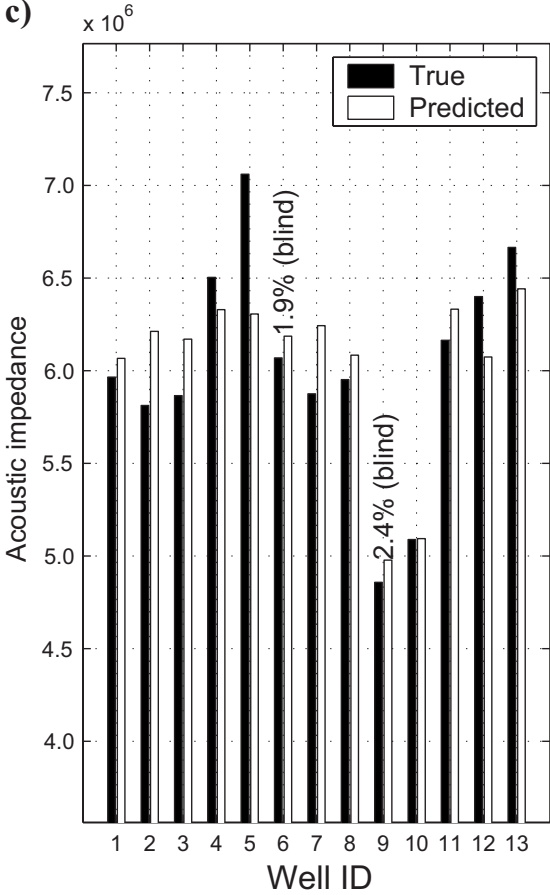

Figure 5. Prediction error at different stages in the learning process: (a) Least-squares linear regression using all attributes. (b) Least-squares linear regression using selected attributes (feature selection). (c) Feature selection and cross validation of the presented technique. Wells 6 and 9 were not used by the learning algorithm. See Table 1 for associated cross-validation errors.

Table 1. Prediction errors (PE) of the used and blind wells (wells 6 and 9) and cross-validation error (CVE) at top chalk for the three cases. (a) Least-squares linear regression. (b) Least-squares linear regression after feature selection. (c) Tikhonov-regularized least squares and cross validation after feature selection. All values are in percent of the mean impedance at the horizon.

\begin{tabular}{lcrrr}
\hline & PE & & PE & PE \\
& (used) & CVE & (w6) & (w9) \\
\hline Regression & 0.0 & 43 & 16.1 & -1.1 \\
Regression + FS & 2.0 & 106 & -5.3 & 3.0 \\
Tikhonov cross validation + FS & 4.4 & 7 & 1.9 & 1.9 \\
\hline
\end{tabular}

Table 2. Prediction errors (PE) of the used and blind wells (well 9) and cross-validation error (CVE) at intra chalk for the three cases. (a) Least-squares linear regression. (b) Least-squares linear regression after feature selection. (c) Tikhonov-regularized least squares and cross validation after feature selection. All values are in percent of the mean impedance at the horizon. Well 6 is not deep enough to penetrate this horizon.

PE (used) CVE PE (w9)

$\begin{array}{llll}\text { Regression } & 0.0 & 51.1 & -6.7\end{array}$

$\begin{array}{llll}\text { Regression + FS } & 0.0 & 56.7 & 35.6\end{array}$

$\begin{array}{llll}\text { Tikhonov cross validation }+ \text { FS } & 3.2 & 4.8 & 4.6\end{array}$ 
a)

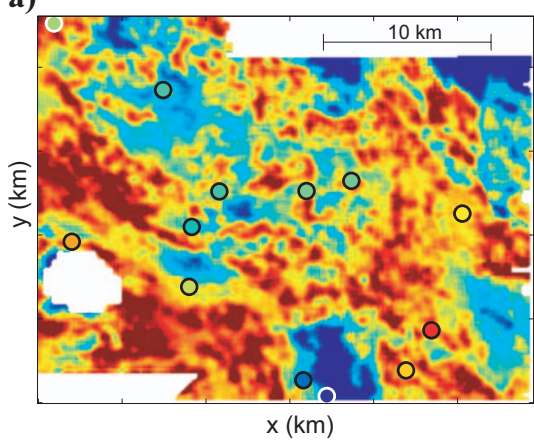

c)

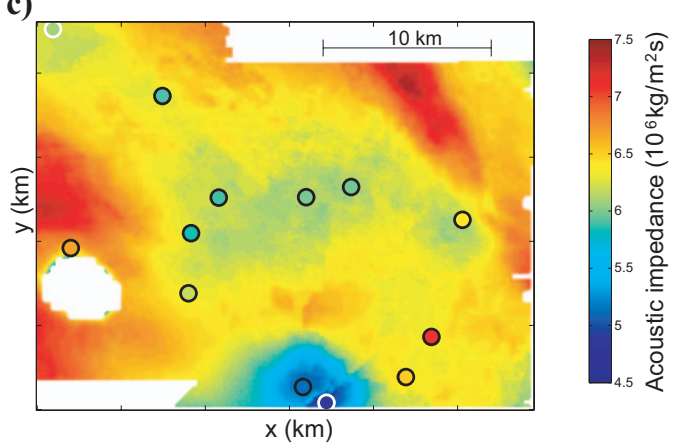

Figure 6. Prediction of the low-frequency impedance along the top chalk horizon in the test area using (a) least squares using all attributes, (b) least squares and feature selection, and (c) feature selection and cross validation of the presented technique. True well-log values are plotted as dots and color coded using the same color scheme as the predicted values. Dots with black borders are used by the learning algorithm, whereas white dots with white borders are blind data. Same color scale is used for all figures. a)

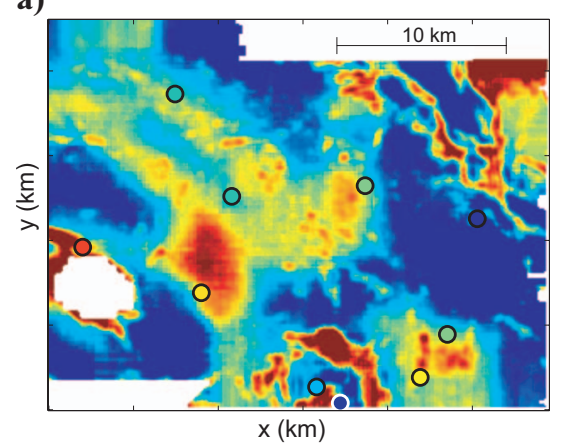

b)

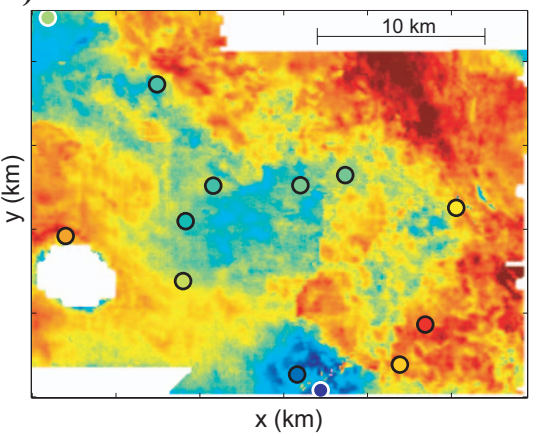

tion covers any approach that reduces the size and complexity of the learning problem, using only the most important attributes.

We applied a simple form of feature extraction based on covariance analysis. Using only attributes with correlation above a certain minimum threshold, the dimension of the attribute space was reduced. In neural network analysis, this approach is used to prevent overfitting of low-frequency impedances.

The test case included five two-way traveltime attributes, and attributes with a correlation above 0.7 to the low-frequency impedance. The correlation level was chosen to remove attributes with little or no correlation. This reduced the number of attributes at the top chalk horizon from 26 to eight. See Table 3.

Figure $5 \mathrm{~b}$ shows the predicted and estimated low-frequency impedance at top chalk using the remaining attributes. Table 1 shows the resulting mean prediction and cross-validation error; see equation 5 . The number of attributes now is fewer than the number of low-frequency observations at the wells, and the prediction error is nonzero. The cross-validation error has increased to $106 \%$, indicating a rather bad result. The prediction error at one well has decreased significantly from about $16 \%$ to $5 \%$, and somewhat increased at the other blind well, indicating no overall improvement. Figure $6 \mathrm{~b}$ illustrates the corresponding prediction at top chalk level, whereas Figure $7 \mathrm{~b}$ shows the prediction of the low-frequency impedance along the intra chalk horizon.
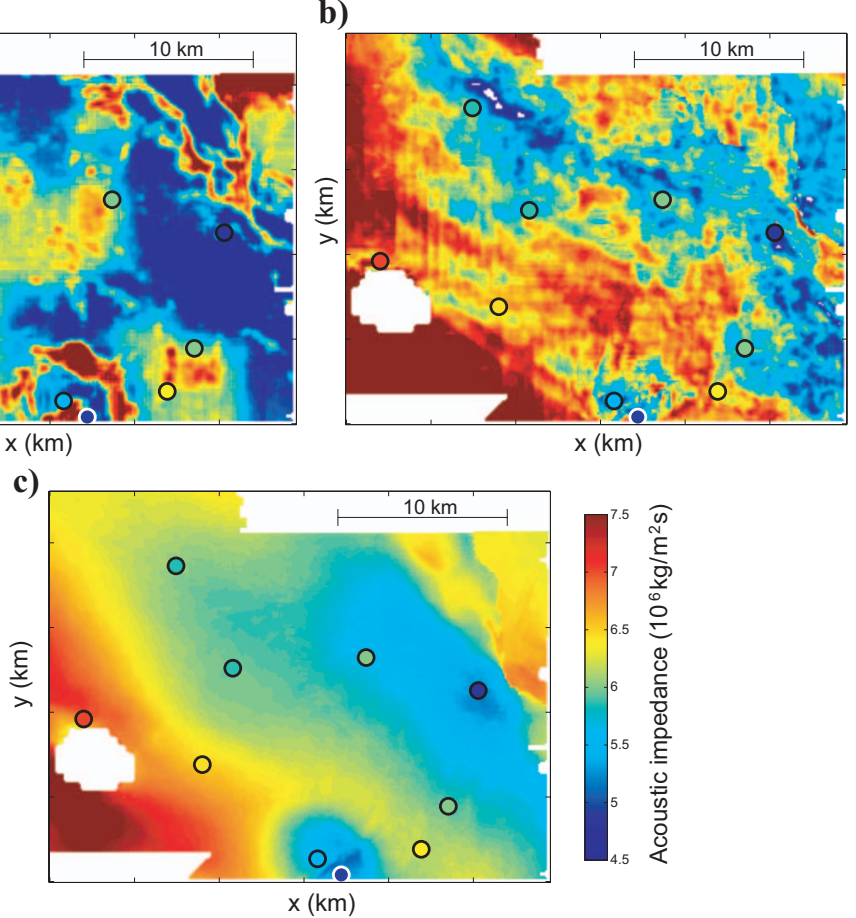

Figure 7. Prediction of the low-frequency impedance along the intra chalk horizon in the test area using (a) least squares using all attributes, (b) least squares and feature selection, and (c) feature selection and cross validation of the presented technique. See Figure 6 for description. Same color scale is used for all figures.

\section{Cross-validated Tikhonov regularization}

In case more attributes are available than welllog data, there exist many solutions that exactly map the well data, but prediction estimates away from the wells most likely will be unstable. The cross-validation approach is designed to eliminate automatically attributes with the lowest multidimensional correlation to observed low-frequency well data.

However, if the number of attributes is much larger than the number of well-log data, the problem of estimating a prediction operator becomes very underdetermined. It is then possible to obtain a very low cross-validation error for extreme combinations of weights and damping factor, even when using the cross-validation strategy (equation 5).

This results in the apparent paradox that wells could be removed one by one from the learning algorithm (equation 3), still giving a perfect prediction result without considering the data. To overcome this problem, we used generalized cross validation, whereby sets of two or more wells were removed to obtain the cross-validation error, as opposed to removing just one (as as- 
sumed in equation 5). In fact, removing just two wells at a time produced very stable results. One drawback of this approach is minimization of the cross-validation error takes more time. Using 13 wells, and removing sets of two wells at a time instead of one, increases the number of blind-well predictions from 13 to $(13 \times 11) / 2=78$, and removing sets of three wells increases the number of blind-well predictions to $(13 \times 12 \times 11) / 6=286$, and therefore increases the CPU power needed by a factor of about 6 and 22 , respectively.

The weights $\mathbf{W}_{m}, \mathbf{W}_{d}$, and the damping factor $\lambda$, are found by minimizing $E_{t}$ in equation 4 . We tested several methods for this minimization and found that a global-search algorithm based on the Metropolis algorithm (Metropolis et al., 1953) provided the best results and was computationally feasible. For this case study, about an hour of CPU time on a simple $3 \mathrm{Ghz}$ Pentium 4 processor was needed. As an example, for the top chalk horizon an optimal damping was found for $\lambda=6.4$, and the corresponding set of optimal weights is listed in Table 3. As can be seen, most dominant are the shallow TWT attributes and the amplitude at top chalk, as well as the interval velocity. Not only do the weights minimize equation 4 , they also produce geologically meaningful results.

Figure 5c shows the estimated and true low-frequency impedance at top chalk using the least-squares solution with Tikhonov regularization, and minimizing the cross-validation error. The prediction error is nonzero (see Tables 1 and 2) for well data used in the learning process, but the cross-validation error is lowered significantly.

Tables 1 and 2 also show that minimizing the expression in equation 4 causes the cross-validation error and the prediction error to be very close. This result is a measure of the quality of the prediction operator and indicates that the method performs equally well away from the wells as at the well positions.

Figures $6 c$ and $7 c$ show the impedance estimate after cross validation at top chalk and intra chalk levels, respectively.

\section{Prediction}

The learning process was performed for all event horizons, estimating the low-frequency impedance for each of them (see, e.g., Figures $6 \mathrm{c}$ and $7 \mathrm{c}$ ), and the uncertainty of the predictions was readily obtained. Figure 8 shows the uncertainty of predictions at top chalk and intra chalk. Note how low uncertainty is not correlated directly to well locations, as would be the case using kriging-based approaches. The observed northwestsoutheast-trending low uncertainty follows a known geologic trend where sedimentary layers are deposited uniformly. Therefore, a low prediction uncertainty should be expected. Note that for the intra chalk horizon, which is not linked to a specific isochron, the uncertainty is higher (Figure $8 b$ ) than for the top chalk horizon (Figure 8a).

\section{Deparameterization}

To deparameterize predicted low-frequency impedance horizons back to an impedance volume, we used vertical 1D interpolation everywhere and applied a 1D vertical low-cut filter resembling the one used in the parameterization process.
Figure $9 \mathrm{a}$ through $9 \mathrm{c}$ shows the profile estimated between the two wells not used by the learning algorithm. Figure $9 \mathrm{a}$ was the result of using the full range of attributes and a simple least-squares regression. Figure $9 \mathrm{~b}$ was the result of using only attributes with high correlation to the low-frequency impedance. Figure $9 \mathrm{c}$ shows the result when the Tikhonov least-squares cross-validation technique was applied. These figures clearly demonstrate the superiority of the crossvalidation approach to conventional regression analysis, as the fit between true and estimated low-frequency logs is excellent using the cross validation and significantly worse using linear regression.

Figure 10 illustrates the predicted profile between wells 4 and 9 after cross validation. The blind well, well 9 , was predicted almost to the accuracy of the input wells (here, well 4), justifying the crossvalidation procedure. Note that some random scattering of the predicted values (the black dots) around the true low-frequency trend was smoothed by the vertical 1D filter. Therefore, the final, predicted low-frequency cube generally is better than results based on singlehorizon predictions.

Table 3. Weights for each of the eight attributes considered at top chalk for Tikhonov-regularized least squares and cross validation after feature selection.

\begin{tabular}{lc} 
Attribute & Log (weight) \\
\hline TWT top chalk & 0.5149 \\
TWT top maast & 3.8452 \\
TWT top hod & 1.0178 \\
TWT intra chalk & 0.1887 \\
TWT base chalk & -0.5501 \\
AMP top chalk & 3.7334 \\
AMP top hod & -0.0651 \\
VEL & 0.8818 \\
\hline
\end{tabular}

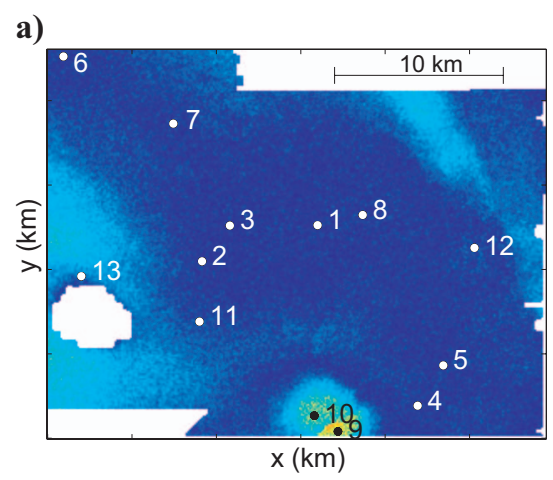

b)

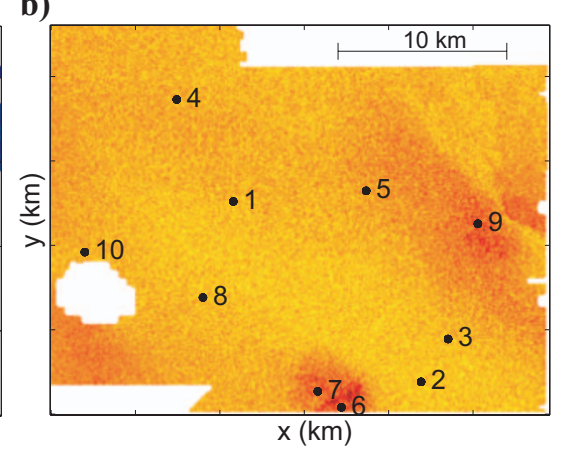

Figure 8. Estimated uncertainty of predictions at (a) top chalk and (b) intra chalk horizons. Blue color indicates relatively low uncertainty $(4.5 \%)$, whereas warmer colors indicate higher uncertainty. Red is the equivalent of $6.0 \%$ uncertainty. The same color scale is used for (a) and (b). 


\section{Effect of each attribute type on the results}

We now consider the results of using each attribute type independently. Figure 11 shows the predicted well logs for well 13 for each attribute type. This well was not used by the learning algorithm. Well 13 is close to the salt dome to the west, and therefore it is an anomaly compared with other wells in the area. Two-way traveltime, thickness, and velocity are the most significant attributes, as shown in Figure $11 \mathrm{a}, \mathrm{b}$ and e. The amplitude and coherency attributes are incapable by themselves of mapping the deepest part of the log correctly (Figure 11c and e).
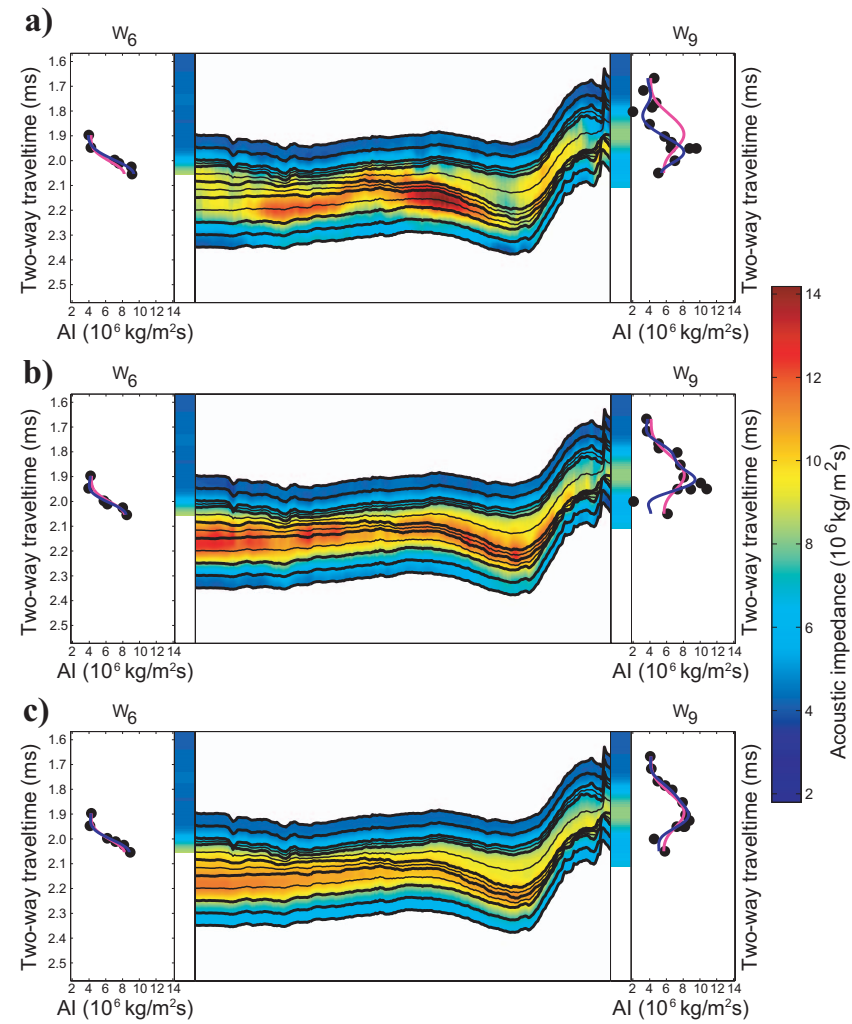

Figure 9. Cross section between well 6 and well 9, neither of which was used by the learning algorithm. (a) Simple least squares. (b) Least squares after feature selection. (c) Feature selection and cross validation of the presented technique. Thin black line is the original well log. Black dots are the impedance estimated at horizons. The purple line is the low-cut-filtered original log. The blue line is the estimated low-cut-filtered impedance log.

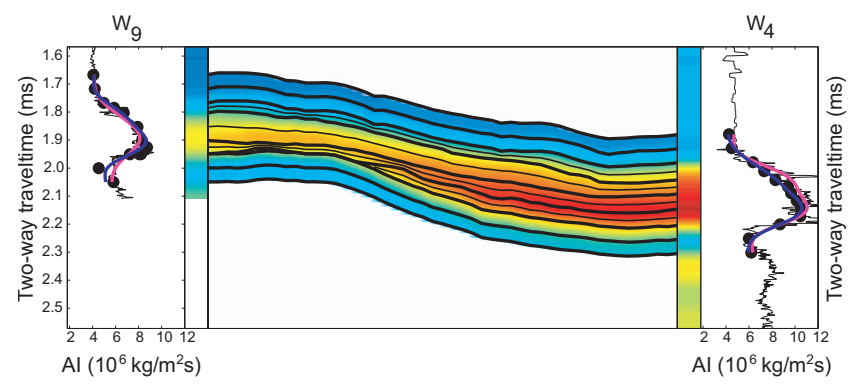

Figure 10. As Figure 9c. Predicted low-frequency impedance profile from well 9 to well 4 . Well 9 was not used in the learning process, whereas well 4 was used. See Figure 9 for line descriptions.
Individual attributes might perform poorly, but linear combinations of attributes might provide better predictions. Well 13 was very well predicted at larger depths, using only traveltime maps (Figure 11a), but less accurately in the upper part of the log. On the other hand, coherence attributes (Figure 11d) produced good predictions at shallow depths but not at larger depths. Figure 12 shows the results of combining two-way traveltime and coherency maps. From this result, it is apparent that the top and lower parts of the log were well predicted.

Such simple combinations of two attributes easily can be investigated manually. It is, however, much harder to find those linear com-

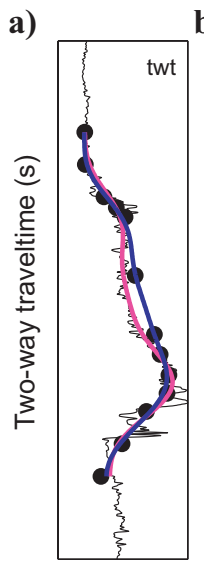

b)

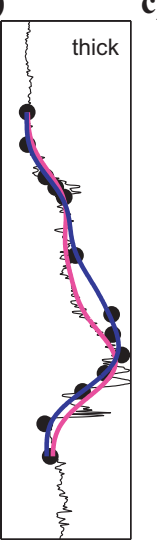

c)

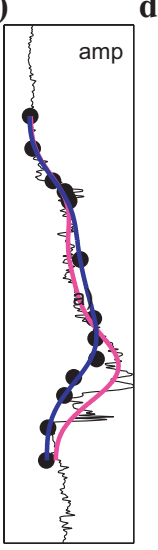

d)

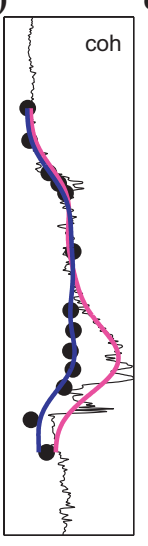

Acoustic impedance $\left(10^{6} \mathrm{~kg} / \mathrm{m}^{2} \mathrm{~s}\right)$ e)

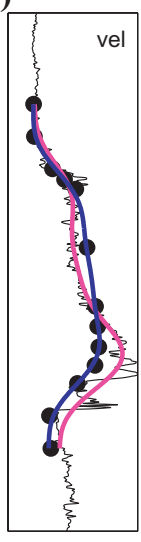

Figure 11. Prediction of blind well 13 using each set of attribute types: (a) two-way traveltime, (b) layer thickness, (c) amplitude, (d) coherency, and (e) chalk velocity. The thin black line is the original well $\log$. Black dots are the impedance estimated at horizons. The purple line is the low-cut-filtered original log. The blue line is the estimated low-cut-filtered impedance log. a)

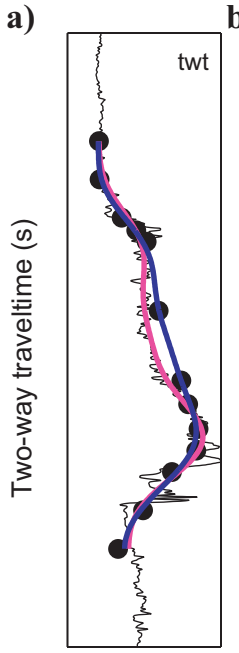

b)

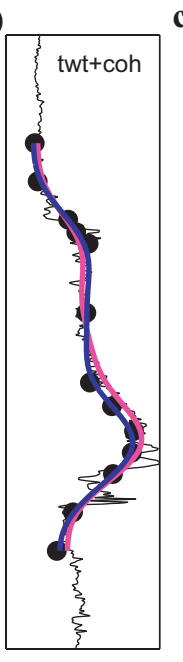

c)

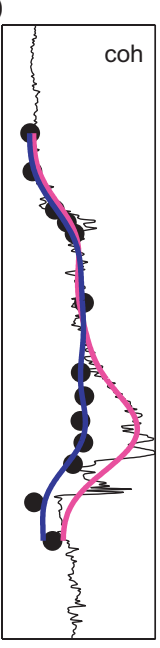

Acoustic impedance $\left(10^{6} \mathrm{~kg} / \mathrm{m}^{2} \mathrm{~s}\right)$

Figure 12. Prediction of blind well 13 using (a) only two-way traveltime, (b) two-way traveltime and coherency, and (c) only coherency. See Figure 11 for description of lines and dots. 
binations of many attributes that give the best results. The cross-validation approach we have chosen aims at finding linear combinations of attributes producing optimal results.

\section{CASE II: DIMENSION REDUCTION AND SPARSE WELL-LOG DATA}

In this study, we investigated the robustness of the cross-validation approach to the number of attributes, and the performance in case of data sparseness.

The investigation involved five impedance well logs, low-cut filtered with a cutoff at $8 \mathrm{~Hz}$, and 15 attributes (six two-way traveltime, five amplitude, and four velocity attributes). The uncertainty of each attribute was defined as in the previous case. Five geologic horizons were used, and in addition, 14 secondary horizons were defined. Figure 13 shows the TWT to the top chalk horizon in the area and the location of the five wells. Initially, we used feature selection based on the correlation approach used previously, and only attributes with a correlation larger than 0.8. A higher threshold for the correlation analysis is chosen than in the previous example because fewer data lead to higher correlation estimates. This reduced the number of attributes to about eight for each horizon. We considered each well as a blind well and made use of the remaining four wells and the attributes to estimate impedance in the blind well. The estimated blind well logs are compared to the true low-frequency well $\operatorname{logs}$ in Figure 14. Generally, the fit between the true and the estimated low-pass-filtered log is excellent, although only four well data were used at each horizon.

Figure 15 illustrates, for comparison, similar predictions with all 15 attributes used by the learning algorithm. The combination of 15 attributes and only four well data leads to a very underdetermined problem. Yet, only minor changes can be observed in the resulting, predicted low-frequency volume. Compare Figures 14 and 15.
This case study suggests that not only is the cross-validation method very robust against the type and number of attributes considered, it is applicable also when well data are sparse.

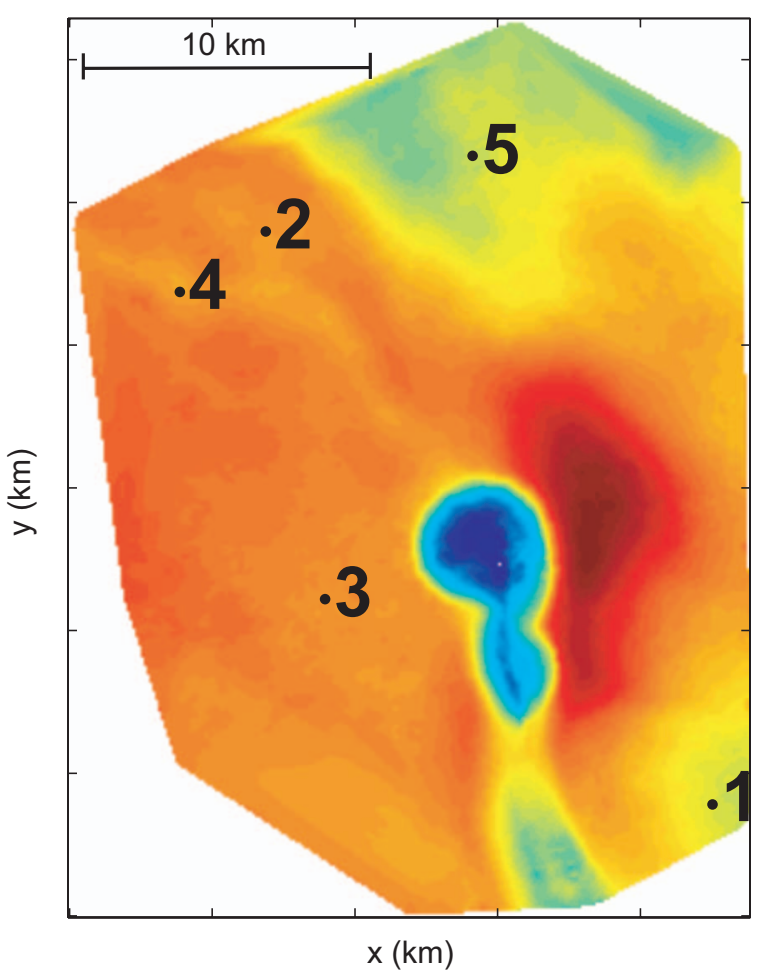

Figure 13. Test area for case II. Five wells available in an area slightly smaller than for case I. Colors reflect the two-way traveltime to top chalk. Blue is for small traveltime, and red is for large traveltime.

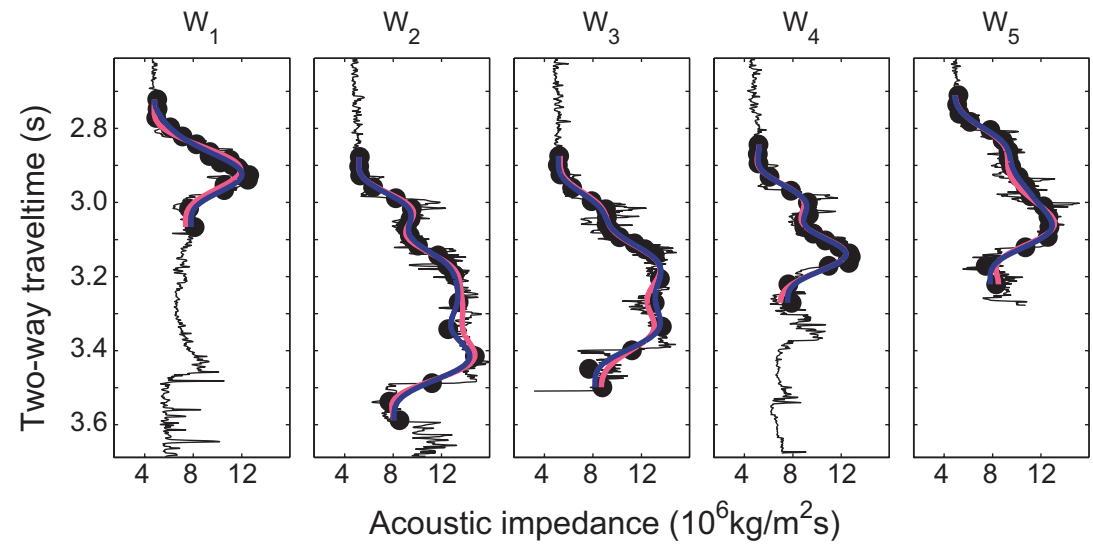

Figure 14. Prediction of each of the five wells when not used in the learning and optimization algorithm. Only attributes with a correlation above 0.8 are used. See Figure 11 for a description of lines and dots. 


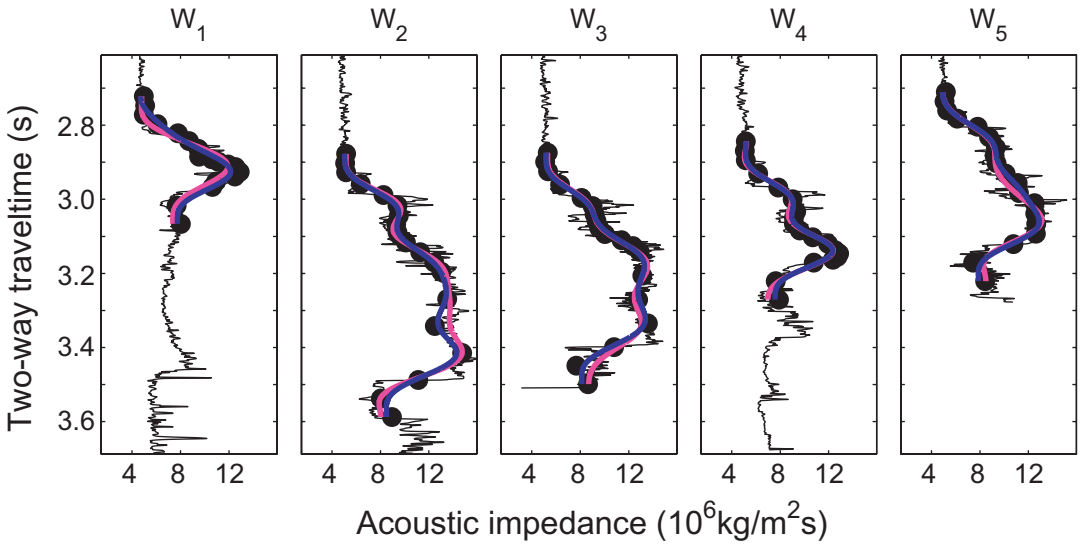

Figure 15. As Figure 14 but when all 17 attributes were used. See Figure 11 for a description of lines and dots.

\section{CONCLUSION}

We have presented a general method based on cross-validated, Tikhonov-regularized, least-squares inversion for multivariate interpolation of sparse direct measurements, guided by dense areacovering attributes.

The method works well, even for highly underdetermined problems (few well-log data and many seismic attributes). The method is fast, as the mapping operator $\mathbf{G}$ can be calculated in a fraction of a second (for the considered data sets) as a function of $\left(\mathbf{W}_{m}, \mathbf{W}_{d}, \lambda, \mathbf{d}, \mathbf{m}\right)$. Therefore, advanced optimization techniques can be used to calculate parameters that minimize the cross-validation error. Uncertainty analysis of input parameters is integrated in the learning and prediction parts of the method. This ensures that attributes resulting in less certain predictions will have less impact than attributes leading to more certain predictions. In addition, the goodness of the prediction operator $\mathbf{G}$ and the interpolated values can be estimated.

We have applied the method to the specific problem of estimating a low-frequency impedance cube from well logs and seismic attributes, using a special parameterization by which the low-frequency impedance is estimated along some two-way traveltime horizons. These 2D maps of low-frequency impedance are combined into a 3D model of low frequency, using vertical interpolation and a vertical low-pass filter.

Numerous examples have shown that this method provides significantly better results than standard linear-regression-based approaches.

The use of cross validation allows for a highly automated method, and for results with the appealing feature that estimations away from boreholes are of the same quality as estimations close to boreholes. This is in contrast to the kriging method that guarantees a perfect well fit at the well location, but away from the well location the trend model controls the impedance estimate. We have demonstrated that using kriging with a trend model to estimate low-frequency impedance can lead to severely distorted results away from well locations. We suggest using the method we present to produce trend models for kriging systems. This will ensure good fit at well locations and stable low-frequency impedance estimation away from well locations.

Case studies have shown that the method we present is very robust against the number of used attributes. In fact, one case study showed that only minimal changes could be noted when going from all at- tributes to the few attributes having the best correlations to low-frequency well-log impedances. This is in contrast to neural networks that typically require some degree of feature selection prior to their application.

The proposed method relies on Gaussian statistics, and hence noncontinuous attributes, such as texture attributes, cannot be used readily. We have shown, however, that inclusion of texture maps, such as coherence maps transformed into fractal dimension, is feasible and valuable as it can lead toward improved predictions.

The method utilizes only linear combinations of attributes, as opposed to neural networks, which can handle strongly nonlinear problems. It is, however, trivial to extend the method to allow nonlinear attribute combinations. This can be done by using a higher-order polynomial fit operator instead of the linear operator used for this study. The current implementation relies on a linear approach because it produced stable results while keeping the required computer power at a reasonable level.

The study area in case study I is part of a much larger area, and the low-frequency impedance is needed for the complete area. Optimizing such a large area might result in less accurate performance for individual wells but also in more stable regional prediction because many different areas are used to produce the same prediction operator. We have investigated the use of cluster analysis to segregate a larger area into smaller areas with similar attribute sets, and the results are promising in conjunction with the proposed method.

Although the examples use 2D interpolation, the theory is not restricted to two dimensions. In principle, a problem of any dimension can be dealt with. Whether the method could be applied directly to estimate the low-frequency impedance in three dimensions, as opposed to the presented $2 \mathrm{D}$ approach, remains to be investigated. One challenge would be the computational efficiency when computing the cross-validation error.

\section{ACKNOWLEDGMENTS}

We thank Mærsk Oil and Gas AS and CORE AS for permission to publish the results of this project. This project was funded jointly by the Danish Agency for Science, Technology and Innovation and COREAS.

\section{REFERENCES}

Banchs, R. E., and J., M. Michelena, 2002, From 3D seismic attributes to pseudo-well-log volumes using neural networks: Practical considerations: The Leading Edge, 21, 996-1001.

Billings, S. D., R. Beatson, and G. Newsam, 2002, Interpolation of geophysical data using continuous global surfaces: Geophysics, 67, 1810-1822.

Bishop, C., 1995, Neural networks for pattern recognition: Oxford University Press.

Brown, A. R., 2001, Understanding seismic attributes: Geophysics, 66, 47-48.

Chopra, S., and K. J. Marfurt, 2005, Seismic attributes - A historical perspective: Geophysics, 70, no. 5, 3SO-28SO.

Claerbout, J. F., 1985, Imaging the earth's interior: Blackwell Scientific Publications.

Doyen, P. M., 1988, Porosity from seismic data - A geostatistical approach: Geophysics, 53, 1263-1275.

Gonzato, G., F. Mulargia, and M. Ciccotti, 2000, Measuring the fractal dimensions of ideal and actual objects: Implications for application in geolo- 
gy and geophysics: Geophysical Journal International, 142, 108-116.

Goovaerts, P., 1997, Geostatistics for natural resources evaluation: Applied Geostatistics Series: Oxford University Press.

Hampson, D., J. Schuelke, and J. Quirein, 2001, Use of multi-attribute transforms to predict $\log$ properties from seismic data: Geophysics, 66, 220-236.

Herrera, V. M., B. Russell, and A. Flores, 2006, Neural networks in reservoir characterization: The Leading Edge, 25, 402-411.

Jannane, M., W. Beydoun, E. Crase, D. Cao, Z. Koren, E. Landa, M. Mendes, A. Pica, M. Noble, G. Roeth, S. Singh, R. Snieder, A. Tarantola, D. Trezeguet, and M. Xie, 1989, Wavelengths of earth structures that can be resolved from seismic reflection data (short note): Geophysics, 54, 906-910.

Kalkomey, C. T., 1997, Potential risks when using seismic attributes as predictors of reservoir properties: The Leading Edge, 16, 247-251.

Leiphart, D. J., and B. Hart, 2001, Comparison of linear regression and a probabilistic neural network to predict porosity from $3 \mathrm{D}$ seismic attributes in Lower Brushy Canyon channeled sandstones, southeast New Mexico: Geophysics, 66, 1349-1358.

Liu, Z., and J. Liu, 1998, Seismic-controlled nonlinear extrapolation of well parameters using neural networks: Geophysics, 63, 2035-2041.

Metropolis, N., A. Rosenbluth, M. Rosenbluth, A. Teller, and E. Teller, 1953, Perspective on equation of state calculations by fast computing machines: Journal of Chemical Physics, 21, 1087-1092.
Neves, F., and S. C. Singh, 1996, Sensitivity study of seismic reflection/refraction data: Geophysical Journal International, 126, 470-476.

Nivlet, P., 2004, Low-frequency constrain in a priori model building for stratigraphic inversion: 74th Annual International Meeting, SEG, Expanded Abstracts, 1802-1805.

Poulton, M., 2002, Neural networks as an intelligence amplification tool: A review of applications: Geophysics, 67, 979-993.

Pramanik, A. G., V. Singh, R. Vig, K. Srivastava, and D. N. Tiwary, 2004, Estimation of effective porosity using geostatistics and multi-attribute transforms: A case study: , 69, 352-372.

Press, W. H., B. P. Flannery, S. A. Teukolsky, and W. T. Vetterling, 1992, Numerical recipes: The art of scientific computing, 2nd ed.: Cambridge University Press.

Russell, B., D. Hampson, T. Todorov, and L. Lines, 2002, Combining geostatistics and multi-attribute transforms: A channel sand case study, Blackfoot oilfield (Alberta): Journal of Petroleum Geology, 21, 97-117.

Tarantola, A., 1987, Inverse problem theory, 4th reprint, 2002: Elsevier.

Tikhonov, A., and V. Arsenin, 1977, Solutions of ill-posed problems: Wiley.

Trappe, H., and C. Hellmich, 2000, Using neural networks to predict porosity thickness from 3D seismic: First Break, 18, 377-384.

Xu, W., T. Tran, R. M. Srivastava, and A. G. Journel, 1992, Integrating seismic data in reservoir modeling: The collocated cokriging alternative: SPE Annual Technical Conference and Exhibition, Paper 24742 